\title{
Cerebral protective mechanisms during cardiovascular surgery: the case of hypothermic circulatory arrest
}

\begin{abstract}
Various surgical procedures require that the normal cerebral blood flow to the brain be interrupted. This challenges surgeons to find means of protecting the brain during these extremely vulnerable periods. Accumulative evidence has shown that the mechanisms underlying neuronal injury is multifactorial, something, which has been attributed to the exceptional complexity of the brain in both, structure and function. As such, efforts to define the best strategies for neuroprotection during circulatory arrest are formidable, at best. Hypothermia has become a cornerstone for cerebral protection during cardiopulmonary bypass (CPB) and is applied to offset the deleterious effects of oxygen deprivation on the brain. The evidence suggests that hypothermia has some action in inhibiting most of injury-inducing processes. Recently, emerging data has indicated that the mechanisms and outcomes of ischemic injury are strongly influenced by biological sex, as well as sex hormones. These observations have further confounded neuroprotection efforts. Despite the various neuroprotective strategies that have recently been introduced, mild hypothermic-circulatory arrest is still commonly used in cardiovascular surgeries. The aim here is to examine the current understanding of the underlying mechanisms of ischemia-induced cerebral injury, to outline the neuroprotective actions of HCA, and finally, briefly outline evidence supporting gender differences in cerebral injury.
\end{abstract}

Keywords: cardiopulmonary bypass, hypothermic circulatory arrest, apoptosis, neural injury, cerebral ischemia, BcL2, BAX, hypothermia, pharmacologic preconditioning
Volume I Issue I - 2015

\author{
Elizabeth $\bigcirc$ Johnson, ${ }^{1,2}$ Theodoros Xanthos ${ }^{2}$ \\ 'Department of Anatomy, National and Kapodistrian University \\ of Athens, Greece \\ ${ }^{2}$ Faculty of Medicine, European University of Cyprus, Cyprus

\begin{abstract}
Correspondence: Elizabeth $\mathrm{O}$ Johnson, Department of Anatomy, National and Kapodistrian University of Athens, School of Medicine, Mikras Asias Str \#75, Goudi I I 527, Athens, Greece, Tel +30 (210) 7462349 , Fax +30 (210) 7462398,620 862I, Email elizabethojohnson@gmail.com
\end{abstract}

Received: September 04, 2015 | Published: October 12, 2015
Abbreviations: $\mathrm{CPB}$, cardiopulmonary bypass; $\mathrm{ABI}$, acquired brain injury; AIF, apoptosis-inducing factor; GABA, gammaaminobutyric acid

\section{Introduction}

Normal blood circulation and adequate oxygenation of the brain and body need to be maintained while surgeons operate on the heart. The ability to maintain blood circulation and oxygenation was achieved with the introduction of cardiopulmonary bypass (CPB). Although $\mathrm{CPB}$ is a pivotal discovery in cardiovascular surgery, it is associated with several morbidities, including morbidity of the kidneys, lungs, and most notably the brain. As such, discovering means to protect the brain during cardiovascular surgery has been the source of focused research. ${ }^{1-7}$ The use of both cardiopulmonary bypass and circulatory arrest have become important means for allowing complex operations on the aorta and brain, and as a means of achieving improved protection of the vulnerable brain. In turn, hypothermic circulatory arrest, which entails cooling the body almost to the point to where it remains in a state of hibernation, has been found to provide the added protection from hypothermia.

Hypothermia has been long been used as a means to protect various organs, including the brain. The first application hypothermia for medicinal purposes dates back to ancient civilizations, most notably the time of Hippocrates. In modern medical history, therapeutic hypothermia was initially described in sparse case reports. Eventually, these initial observations were supported by formal experimental studies in animals. These studies were ultimately followed by the application of hypothermia during circulatory arrest in a controlled clinical setting. ${ }^{8}$ For the most part, both animal and human data advocate that mild hypothermia is beneficial after global cerebral ischemia and reperfusion. ${ }^{1,9}$ A more profound hypothermia (often referred to as deep hypothermic cardiopulmonary arrest) has also been applied to complex surgeries on the brain, heart and aorta. ${ }^{10}$

While HCA is now broadly used in many cardiothoracic, as well as neurosurgical procedures, the best means to achieve effective cerebral protection remains an issue of debate..$^{1-7,9}$ Despite the surge and proposal of various neuroprotective strategies, mild hypothermiccirculatory arrest is still commonly used in cardiovascular procedures. The aim here is to examine the current understanding of the underlying mechanisms of ischemia-induced cerebral injury, to outline the neuroprotective actions of HCA on the brain, and finally, address the evidence supporting gender differences in both cerebral injury and neuroprotective mechanisms.

\section{Cerebral injury}

Various types of brain injuries are now included in the relatively new medical diagnostic category, Acquired Brain Injury (ABI). ABI has a very large scope and reflects any brain injury that is acquired after birth. ${ }^{11}$ The most common etiologies of acquired brain injuries include: vascular disruption, traumatic brain injuries and oxygen deprivation, which can vary significantly in severity and outcomes. Brain injuries from vascular disruption are frequently attributed to cardiovascular 
surgery, heart attaches, stroke, aneurysm, and intracranial surgery, while traumatic brain injuries are typically associated with traffic accidents, assaults, sports accidents. Cerebral injury from oxygen deprivation can be attributed to an obstructed airway, asthma, birth complications and severe chest injuries. Additionally, it should be noted that cerebral injury might also be attributed to various other processes, including neurodegenerative diseases, systemic disorders or infectious diseases, among others.

Despite dedicated efforts to define means of neuroprotection during surgery, acquired brain injury following cardiovascular procedures is unacceptably high, with almost $80 \%$ of the patients demonstrating some form of neuropsychological deficit after cardiac surgery. The reported incidence of cognitive deficits at the time of discharge varies from $45 \%$ to $88 \%$, which decreases only slightly after about six weeks (between $15 \%$ and $36 \%$ ). ${ }^{12-15}$ While the aim of HCA was to prevent cerebral ischemia and hence, neurologic injury, neuropsychological deficits remain a common outcome following its use. Close to $30 \%$ of the patients undergoing HCA present with a transient neurologic deficit, while almost $14 \%$ present with a permanent cerebral deficit. The significance of cerebral injury following HCA is underscored by the observation that among those patients undergoing HCA who sustain neurologic injury, early postoperative mortality significantly increases to close to $20 \%{ }^{16-23}$

The extent and severity of cerebral injury following cardiopulmonary bypass and HCA has prompted a deluge of experimental and clinical studies aimed at addressing the mechanisms underlying the neuronal injury process, as well as those underlying the neuroprotection afforded by hypothermia. Cerebral ischemia, such as that induced during HCA, is able to promote cascadse of events, including those related to cell-death pathways, inflammation, disruption of electrolyte homeostasis, among others. Activation of any of the processes can culminate in cell dysfunction, and ultimately cell death.

Several studies have demonstrated that the neurologic injury following cardiopulmonary bypass may be attributed to the induction of cell-death pathways, including cellular necrosis and apoptosis, as a result of global cerebral ischemia. ${ }^{24-26}$ Both types of cell death, although believed by some to be part of a biological continuum, have notable differences in both the morphological features expressed by the nerve cells, as well as by the molecular cascade of events which orchestrates the death process. ${ }^{27}$ Edema and inflammation are central components of the apparently irreversable and uncontrolled process of necrosis, where complete energy failure ultimately leads to the complete lysis of the cell

Apoptosis, on the other hand, involves a tightly controlled series of molecular events. Apoptotic cell death entails an interplay between pro-apoptotic and anti-apoptotic proteins, induced by enzymatic pathways. This protein interplay is considered a critical component of the "decision phase", which ultimately determines whether the cell will survive or progress to cell death. Proteins from the Bcl-2 family are the primary players at this critical intracellular decision checkpoint; the induction of these proteins are controlled by the the Bcl-2 and p53 genes. ${ }^{28}$ Anti-apoptotic proteins, including Bcl2 and $\mathrm{Bcl}-\mathrm{xL}$, act to inhibit apoptosis and protect the neuron from committing suicide. On the other hand, pro-apoptotic proteins, including Bax, Bak and Bcl-xs, promote apoptosis, hence acting to induce cell death. ${ }^{28}$ Current evidence suggests that it is the ratio of the anti-apoptotic (e.g., Bcl-2) to pro-apoptotic (e.g., Bax) proteins, which are induced after an ischemic insult that ultimately determines whether the nerve cell will proceed to cell death or will survive. ${ }^{29}$ This hypothesis has been supported in controled experimental studies, where increased expresion of Bcl-2 was associated with increase cell survival following ischemic insult in an acute porcine model. ${ }^{5,6}$

Ultimately, if the release of pro-apoptotic proteins (e.g., Bax) is greater than the protective $\mathrm{Bcl}-2$ proteins, the nerve cell will ultimately progress to the "execution stage" of the cell death program. The elevated levels of Bax like proteins, stimultes the activation of caspases. Caspases, in turn, promote the induction of a proteolytic cascade that disrupts the structural and functional integrity of the cell, which includes the fragmentation of the DNA. ${ }^{28,30,31}$ Assaying for the presence of DNA fragmentation using the Tunel assay, has become one of the hallmarks used by investigators for determining apoptotic cell death. ${ }^{32,33}$

Cell death cascades are also associated with significant disruption of critical cell homeostatic mechanisms, which have been attributed to the lack of oxygen. For example, neuronal injury is associated with marked disruption of the balance between energy depletion-waste product accumulation. The molecular process of cell death is also associated with the imbalance in calcium levels induced by the release of excitatory neurotransmitters that activate ion channels leading. ${ }^{34,35}$

Both acute and prolonged inflammatory processes play important roles in the induction of cerebral damage following ischemic insult. These processes are characterized by rapid activation of resident cells, production of proinflammatory mediators, such as cytokines, and infiltration of of inflammatory cells. All of these time-dependent processes can induce significant injury to the brain. Cytokines are important molecular signals in the inflammatory response to cerebral ischemia. Hence, with the onset of circulatory arrest various cytokines and complement anaphylatoxins are released leading to chemotaxis of polymorphonuclear leukocytes expression of adhesion molecules, increased vascular permeability, activation of blood coagulation and platelet activation. ${ }^{36}$ Moreover, release of reactive oxygen species and cytokines enhance both endothelial damage and release of vasoactive substances, which eventually cause various degrees of vasoconstriction. ${ }^{37}$

At the microcirculatory level, arterioles are richly adrenergically innervated, thus responding to adrenergic stimulation causing vasoconstriction. Following sympathetic stimulation, the A1 and A2 arterioles show the greatest percentage of change in diameter values and remain constricted for long periods of time, whereas the A3 and A4 arterioles respond initially via constriction, but return to their initial diameter in a short period of time..$^{38}$ In addition intracellular acidosis occurs within seconds of circulatory arrest leading to rapid ATP depletion and lactate accumulation. Consecutively, the blood brain barrier of various brain regions breaks down at a very selective and specific manner. ${ }^{39}$

At the same time, the damage of the fatty acids of the cell membrane, which increases membrane permeability, leads to severe derangement of intracellular electrolytes. ${ }^{18,19,40}$ Studies have shown that the passage of large molecules through the blood brain barrier enhances the passage of $\mathrm{Na}^{+}$from the blood to the brain altering thus the osmolality and they result in cell swelling and brain edema. ${ }^{37}$ The end effect is selective neuronal damage and widening of extracellular spaces within the brain. ${ }^{41}$ This may displace neuronal, glial, and endothelial cell interaction, damaging, thus, neurophil and synaptic structures and/or contacts. ${ }^{36}$ 
It is intriguing that studies have shown that certain areas of the brain appear to be more susceptible to neurologic damage following the mild ischemic insult induced by HCA. ${ }^{42,43}$ Cerebral injury following mild global ischemia induced in these studies was expressed as either disruption of the normal neuronal cell structure, or fragmentation of the DNA. In turn, it appears that specific neurologic deficits, such as sensory-motor function and cognition, may be attributed, at least in part, to the apparent selective morbidity of specific neuronal areas. ${ }^{44,45}$ In this regard, the sensory and motor neocortex were reported as particularly sensitive areas to ischemic injury in the brain. ${ }^{42}$ In addition the these particular areas showing an increased vulnerability to ischemic insults, these same well-defined neuronal areas also showed an increased responsiveness to neuroprotective strategies. ${ }^{5,6}$

\section{Neuroprotection}

Today, hypothermia remains one of the most widely used methods for neuroprotection. One of the most central premises behind the application of hypothermia for neuroprotection is its effectiveness in reducing the brain's rate of metabolism. ${ }^{46}$ The central nervous system receives about $15 \%$ of the cardiac output and consumes $20 \%$ of the oxygen required by the body at rest. The oxygen consumption is approximately $3.5 \mathrm{ml} / 100 \mathrm{gr}$ brain tissue/min, indicating an exceptionally high metabolic rate. ${ }^{47}$ Additionally, the brain has an accelerated rate of glucose consumption/metabolism, which provides the primary source of neuronal energy and are strictly oxygendependent mechanisms. Cerebral blood flow decreases linearly with reductions in temperature. In addition, hypothermia also decreases the brains metabolic rate, hence significantly decreasing the need for both oxygen and glucose. ${ }^{48}$ In contrast, linear association between cerebral blood flow and temperature reduction, $\mathrm{CMRO}_{2}$ decreases exponentially with reductions in temperature. ${ }^{49}$

In addition to lowering the brains requirement for oxygen and glucose by decreasing the rate of metabolism, hypothermia also reduces the release of excitatory neurotransmitters, which in turn result in the decrease the calcium uptake by nerve cells. Prevention of calcium ion uptake by nerve cells inhibits the activation of intracellular proteases, as well as dysfunction of the mitochondria, which could promote irreversible nerve cell injury or cell death. ${ }^{35}$ Hypothermia also reduces inflammatory cytokines that are released with the onset of circulatory arrest. ${ }^{37}$ The inflammatory mechanisms induced by cerebral ischemia may last for an extended period. Hypothermic-circulatory arrest decreases both the production and release of cytokines, as well as modulates the function of inflammatory cells.

A protective mechanism of hypothermia that has received much interest is its potential to inhibit of neuronal apoptosis. This hypothesis has been supported by studies showing fewer histological disruptions, ${ }^{1}$ and less disruption of the DNA integrity. ${ }^{5,6}$ In addition to a significant decrease in the traditional hallmarks of apoptosis, particularly DNA fragmentation, hypothermia was also associated with an increase in anti-apoptotic proteins in areas of the brain that were previously found to be selectively vulnerable. ${ }^{5,6}$ Taken together, the data suggests that hypothermia may improve neurological outcome, at least in part, by inhibiting the apoptotic biochemical cascade.

The decreased sensitivity of brain to ischemic injury with hypothermia, has suggested to some researchers and clinicians that profound hypothermia may afford even greater neuroprotection, although the apparent clinical application seems intuitively limited. ${ }^{1,2,50-52}$ In this regard, cerebral oxygen metabolism is significantly reduced with profound hypothermia at $8 \mathrm{oC}$, while at
$18 \mathrm{oC}$ it remains as high as $24 \%$ of baseline, suggesting a less complete cerebral protection at the latter temperature. In our acute piglet model, we have reported that profound hypothermia at $10 \mathrm{oC}$ is associated with significant reduction of apoptotic cells in the neocortex. ${ }^{5,6}$

Despite this apparent benefit, deep hypothermia requires longer cardiopulmonary bypass times and this entails the consumption of clotting factors that may result in coagulation disturbances. Deep hypothermia has also been shown to impair platelet activity and reduce the enzymatic activity of clotting factors upon the activation of coagulation. ${ }^{50,51}$ However, hypothermia exerts its neuroprotective effect when profound, though not easily and safely clinically applicable, and our experimental study is a proof of this concept.

Risk factors associated with less favorable cerebral outcome after circulatory arrest includes hyperglycemia either before arrest or during reperfusion. The increased brain damage in the presence of hyperglycemia is related to increased production of lactate and to the accompanying increase in tissue acidosis. In our study, lactate levels were measured, as it is known that blood glucose and lactate are interrelated via the pathway of anaerobic glycolysis. It is not clear why higher lactate levels were reported in the cooling and significantly in the rewarming phase of the $10^{\circ} \mathrm{C}$ treated animals. Because the brain's blood flow is significantly reduced during deep hypothermia, the accumulation of lactate may be reduced as a result of the decreased substrate for glucose metabolism. ${ }^{46}$

\section{Gender-specific neuronal vulnerability and neuroprotection}

Researchers are aware of the cascade of pathologic events triggered by cerebral ischemia that culminate in cell dysfunction and death. While the last decade has focused on a molecular dissection of the pathways involved, attempts at finding effective means of neuroprotection have largely been unsuccessful. This may be explained, in part, by the emerging evidence of gender differences in various pivotal points of the activated molecular pathways.

Stemming from epidemiologic data, the concept that the mechanisms and outcome of ischemic injury are strongly influenced by biological sex has emerged. ${ }^{53}$ Premenopausal women have a lower incidence of stroke compared to men and a better recovery after traumatic brain injury. ${ }^{54}$ Evidence has accumulated in recent years indicating the molecular cascades activated by cerebral ischemia are not the same in neurons of females and males. This has been supported by both studies in cell cultures, and animal studies.

Sex hormones were initially believed to be the major contributor to sex differences observed. Both progesterone and estrogen have been associated with neuroprotective attributes. ${ }^{55}$ Various neuroprotective mechanisms have been attributed to progesterone including modulation of gamma-aminobutyric acid (GABA) and enhancement of GABA ergic neurotransmission, which appears to counter neuroexcitoxicity. In addition, progesterone appears to inhibit apoptosis ${ }^{56}$ and the production of inflammatory mediators. ${ }^{57}$ Estrogen, on the other hand, is believed to function as an antioxidant, protecting against glutamate-induced excitotoxcity and promoting prosurvival gene expression. ${ }^{53}$ In addition to biologic sex and gonadal steroids, current evidence also supports that brain-derived neurosteroids may play a role as modulators of ischemic neurologic damage. Neurosteroids are defined as steroid hormones that are synthesized locally within the brain. 
In addition to the direct effects of sex hormones, sex differences may manifest as the result of effects mediated by the sex chromosomes or even in the absence of hormone exposure, as a result of the organizational effects of sex hormones during development. ${ }^{58}$ On the other hand, several differences in the brain's response to ischemia between the genders appear to be independent of hormonal action, and dependent on innate neuronal differences. ${ }^{58}$ Two signaling cascades have been identified in apoptosis; one dependent on capsize activity (caspase-dependent pathway), and one dependent upon apoptosis-inducing factor (AIF) (caspase-independent pathway). ${ }^{59,60}$ It appears that females are exquisitely sensitive to caspase-mediated cell death, whereas cell death in males is triggered by caspaseindependent pathways. The fact that several clinically relevant neuroprotective agents (e.g., erythropoitetin, hypothermia, caspase inhibitors) have shown clear sexual dimorphic responses, stress the importance of investigating these sex differences further. ${ }^{61}$ Current findings support that some forms of ischemia induced neuronal cell death are gender-specific. In turn, these differences strongly suggest that neuroprotective strategies will also demonstrate gender-specific effectiveness. At present, there is limited to no data indicating whether there is a dimorphic response to hypothermic circulatory arrest. The ability to devise novel and reliable neuroprotecitve strategies will need to understand and take into account the underlying mechanisms of neuronal injury and their potential sex differences. ${ }^{62-66}$

\section{Conclusion}

In conclusion, although there is evidence of some cerebral injury following hypothermic circulatory arrest, it still remains an effective neuroprotective strategy during complex cardiovascular procedures. Studies suggest that hypothermia may be able to promote neuroprotection and act at various levels, including inhibiting the molecular cascades responsible for controlled cell death, inflammation, and cellular homeostatic mechanisms among others. Recent evidence shows a sexual dimorphism in the brain's response to ischemic injury, although there is no evidence yet that supports gender differences in HCA-induced mild global ischemia. Taken together, moderate hypothermic circulatory arrest still remains an important part of the surgeon's armamentarium.

\section{Acknowledgements}

None.

\section{Conflict of interest}

The author declares no conflict of interest.

\section{References}

1. McCullough JN, Zhang N, Reich DL, et al. Cerebral metabolic suppression during hypothermic circulatory arrest in humans. Ann Thorac Surg. 1999;67(6):1895-1899.

2. Ehlrich MP, McCullough JN, Zhang N, et al. Effect of hypothermia on cerebral blood flow and metabolism in the pig. Ann Thorac Surg. 2002;73(1):191-197.

3. Strauch JT, Spielvogel D, Haldenwang PL, et al. Cooling to $10^{\circ} \mathrm{C}$ and treatment with cyclosporine A improve cerebral recovery following prolonged hypothermic circulatory arrest in a chronic porcine model. Eur $J$ Cardiothorac Surg. 2005;27(1):74-80.

4. Grigore AM, Murray CF, Ramakrishna H, et al. A core review of temperature regimens and neuroprotection during cardiopulmonary bypass: does rewarming rate matter. Anesth Analg. 2009;109(6):1741-1751
5. Ananiadou OG, Bibou K, Drossos GE, et al. Effect of profound hypothermia during circulatory arrest on neurologic injury and apoptotic repressor protein BcL-2 expression in an acute porcine model. $J$ Thorac Cardiovas Surg. 2007;133(4):919-926.

6. Ananiadou OG, Bibou K, Drossos GE, et al. Hypothermia at 10C reduces neurologic injury after hypothermic circulatory arrest in the pig. $J$ Cardiac Surg. 2008;23(1):31-38.

7. Brambrink AM, Koerner IP, Diehl K, et al. The antibiotic erythromycin induces tolerance against transient global cerebral ischemia in rats (pharmacologic preconditioning). Anesthesiology. 2006;104(6):1208-1215.

8. Apostolakis E, Shuhaiber JH. Antegrade or retrograde cerebral perfusion as an adjunct during hypothermic circulatory arrest for aortic arch surgery. Semin Cardiothoracic Vasc Anesth. 2007;11(1):77-85.

9. Froehler MT, Geocadin RG. Hypothermia for neuroprotection after cardiac arrest: mechanisms, clinical trials and patient care. J Neurol Sci. 2007;261(1-2):118-126

10. Griepp RB1, Ergin MA, McCullough JN, et al. Use of hypothermic circulatory arrest for cerebral protection during aortic surgery. J Card Surg. 1997;12(2 suppl):312-321.

11. Finnerty F, Glyn L, Dineen B, et al. A postal surfey of data in general practice on the prevalence of acquired brain injury $(\mathrm{ABI})$ in pateints aged 18-65 in one county in the west of Ireland. BMC Family Pract. 2009; $10: 36-42$.

12. Djaiani G, Fedorko L, Borger MA, et al. Continuous-flow cell saver reduces cognitive decline in elderly patients after coronary bypass surgery. Circulation. 2007;116(17):1888-1895.

13. Ho PM, Arciniegas DB, Grisgsby J, et al. Predictors of cognitive decline following coronary artery bypass graft surgery. Ann Thorac Surg. 2004;77(2):597-603

14. Neville MJ, Butterworth J, James RL, et al. Similar neurobehavioral outcome after valve or coronary arteryoperations despite differing carotid embolic counts. J Thorac Cardiovasc Surg. 2001;121(1):125-136.

15. Ricotta JJ, Faggioli GL, Castilone A, et al. Risk factors for stroke after cardiac surgery: Buffalo cardiac-cerebral study group. J Vasc Surg. $1995 ; 21(2): 359-363$.

16. Coselli JS, Bozinovski J, Cheung C. Hypothermic circulatory arrest: safety and efficacy in the operative treatment of descending and thoracoabdominal aortic aneurysms. Ann Thorac Surg. 2008;85(3):956-964.

17. Hickey PR. Neurologic sequlae associated with deep hypothermic circulatory arrest. Ann Thorac Surg. 1998;65(6 suppl):S65-70.

18. Nollert G, Nagashima M, Bucerius J, et al. Oxygenation strategy and neurologic damage after deep hypothermic circulatory arrest. Hypoxia versus free radial injury. J Thorac Cardiovasc Surg. 1999;117(6):11721179.

19. Reich DL, Uysal S, Sliwinski M, et al. Neuropsychologic outcome after deep hypothermic circulatory arrest in adults. J Thorac Cariovasc Surg. 1999;117(1):156-163.

20. Baumgartner WA, Walinsky PL, et al. Assessing the impact of cerebral injury after cardiac surgery: will determining the mechanism reduce this injury? Ann Thorac Surg. 1999;67(6):1871-1873.

21. Cook DJ, Huston J, Trenerry MR, et al. Postcardiac surgical cognitive impairment in the aged using diffusion-weighted magnetic resonance imaging. Ann Thorac Surg. 2007;83(4):1389-1395.

22. Hirsch JC, Jacobs ML, Andropoulos D, et al. Protecting the infant brain during cardiac surgery: a systematic review. Ann Thorac Surg. 2012;94(4):1365-1373. 
23. Knipp SC, Matatko N, Wilhelm H, et al. Cognitive outcomes three years after coronary artery bypass surgery: relation to diffusion-weighted magnetic resonance imaging. Ann Thorac Surg. 2008;85(3):872-879.

24. Hagl C, Tatton NA, Khaladj N, et al. Involvement of apoptosis in neurological injury after hypothermic circulatory arrest: a new target for therapeutic interventions? Ann Thorac Surg. 2001;72(5):1457-1464.

25. Brock MV1, Blue ME, Lowenstein CJ, et al. Induction of neuronal nitric oxide following hypothermic circulatory arrest. Ann Thorac Surg. 1996;62(5):1313-1320.

26. Bottiger BW, Schmitz B, Wiessner C, et al. Neuronal stress response and neuronal cell damage after cardiocirculatory arrest in rats. J Cereb Blood Flow Metab. 1998;18(10):1077-1087.

27. Kerr JF, Wyllie AH, Currie AR. Apoptosis: A basic biological phenomenon with wide ranging implication in tissue kinetics. $\mathrm{Br} J$ Cancer. 1972;26(4):239-257.

28. Kam PC, Ferch NI. Apoptosis: mechanisms and clinical implications. Anaesthesia. 2000;55(11):1081-1093.

29. Yang J, Liu X, Bhalla K, et al. Prevention of apoptosis by Bcl2 release of cytochrome $\mathrm{C}$ from mitochondria blocked. Science. 1997;275(5303):1129-1132.

30. Ashraf QM, Zubrow AB, Mishra OP, et al. Nitration of Bax and Bcl-2 proteins during hypoxia in cerebral cortex of newborn piglets and the effect of nitric oxide synthase inhibition. Biol Neonate. 2002;81(1):65-72.

31. Lo AC, Houenou LJ, Oppenheim RW. Apoptosis in the nervous system: morphological features, methods, pathology, and prevention. Arch Histol Cytol. 1995;58(2):139-149.

32. Martin LJ. Neuronal cell death in nervous system development, disease and injury. Int J Mol Med. 2001;7(5):455-478.

33. Sastry PS, Kalluri SR. Apoptosis and the nervous system. J Neurochem. 2000;74(1):1-20.

34. Gunn AJ, Thoresen M. Hypothermic neuroprotection. NeuroRx. 2006;3(2):154-169.

35. Gonzalex-Ibarra FP, Varon J, Lopez-Meza EG. Therapeutic hypothermia: critical review oft he molecular mechanisms of action. Front $\mathrm{Neu}$ rol. 2011;2:4.

36. Chalkias A, Xanthos T. Post-cardiac arrest brain injury: Pathophysiology and treatment. J Neurol Sci. 2012;315(1-2):1-8.

37. Sharma HS, Miclescu A, Wiklund L. Cardiac arrest-induced regional blood-brain barrier breakdown, edema formation and brain pathology: a light and electron microscopic study on a new model for neurodegeneration and neuroprotection in porcine brain. J Neural Transm. 2011;118(1):87-114.

38. Xanthos T, Chalkias A. Therapeutic hypothermia: Focus on microcirculation. Resuscitation. 2014;85(5): 583-584.

39. Muresanu DF, Sharma A, Sharma HS. Diabetes aggravates heat stress-induced blood-brain barrier breakdown, reduction in cerebral blood flow, edema formation, and brain pathology: possible neuroprotection with growth hormone. Ann N Y Acad Sci. 2010;1199:15-26.

40. Sharma AB, Sun J, Howard LL, et al. Oxidative stress reversibly inactivates myocardial enzymes during cardiac arrest. Am J Physiol Heart Circ Physiol. 2007;292(1):H198-206.

41. Castejón OJ. The extracellular space in the edematous human cerebral cortex: an electron microscopic study using cortical biopsies. Ultrastruct Pathol. 2009;33(3):102-111.
42. Ananiadou GO, Drossos EG, Bibou NK, et al. Acute regional neuronal injury following hypothermic circulatory arrest in a porcine model. Interact CardioVasc Thorac Surg. 2005;4(6):597-601.

43. Kurth CD, Priestley M, Golden J, et al. Regional patterns of neuronal death after deep hypothermic circulatory arrest in newborn pigs. $J$ Thorac Cardiovasc Surg. 1999;118(6):1068-1077.

44. Kunihara T, Grün T, Aicher D, et al. Hypothermic circulatory arrest is not a risk factor for neurologic morbidity in aortic surgery: A propensity score analysis. J Thorac Cardiovasc Surg. 2005;130(3):712-718.

45. Lipton P. Ischemic cell death in brain neurons. Physiol Rev. 1999;79(4):1431-1568.

46. Kirklin JW, Barratt-Boyes BG. Cardiac Surgery. Churchill Livingstone 3rd ed. 2003;1:71-72.

47. Clarke DD, Sokoloff L. Circulation and energy metabolism of the brain. In: Siegel GJ editors. Basic neurochemistry molecular, cellular, and medical aspects. 6th ed. Philadelphia, New York, USA: Lippincott Williams \& Wilkins; 1999. p. 637-670.

48. Erecinska M, Thoresen M, Silver IA. Effects of hypothermia on energy metabolism in Mammalian central nervous system. J Cereb Blood Flow Metab. 2003;23(5):513-530.

49. Greeley WJ, Kern FH, Meliones JN, et al. Effect of deep hypothermia and circulatory arrest on cerebral blood flow and metabolism. Ann Thorac Surg. 1993;56(6):1464-1466.

50. Ramsi P, Heikkinen J, Biancari F, et al. Prolonged mild hypothermia after experimental hypothermic circulatory arrest in a chronic porcine model. J Thorac Cardiovasc Surg. 2002;123(4):724-734.

51. Undar A, Vaughn WK, Calhoon JH. The effects of cardiopulmonary bypass and deep hypothermic circulatory arrest on blood viscoelasticity and cerebral blood flow in a neonatal pilet model. Perfusion. 2000;15(2):121-128

52. Hagl C, Khaladj N, Karck M, et al. Hypothermic circulatory arrest during ascending and aortic arch surgery: the theoretical impact of different cerebral perfusion techniques and other methods of cerebral protection. Eur J Cardio-thoracic Surg. 2003;24(3):371-378.

53. Roof RL, Hall ED. Gender differences in acute cns trauma and a stroke: neuroprotective effects of estrogens and progesterone. J Neurotrauma. 2000;17(5):367-388

54. Groswasser Z, Dohen M, Keren O. Female TBI patients recover better than males. Brain Inj. 1998;12(9):805-812.

55. Rogers E, Wagner AK. Gender, sex steroids, and neuroprotection floowing traumatic brain injury. J Head Trauma Rehabtl. 2006;21(3):279281.

56. He J, Hoffman SW, Stein DG. Allopregnanolone, a progesterone metabolite, enhances behavioral recovery and decreases neuronal loss after traumatic brain injury. Restor Neurol Neurosci. 2004;22(1):19-31.

57. Djebaili M, Guo Q, Pettus EH, et al. The neurosteroids progesterone and allopregnanolone reduce cell death, gliosis, and functional deficits after traumatic brain injury in rats. J Neurotrauma. 2005;22(1):106-118

58. Siegel C, Turzo C, McCullough LD. Sex differences in cerebral ischemia: possible molecular mechanisms. J Neurosci Res. 2010;88(13):27652774.

59. Liu F, Li Z, Li J, et al. Sex differences in caspase activation after stroke. Stroke. 2009;40(5):1842-1848.

60. Zhu C, Xu F, Wang X, et al. Different apoptotic mechanisms are activated in male and female brains after neonatal hypoxia-ischemia. J Neurochem. 2006;96(4):1016-1027. 
61. Gibson CL. Cerebral ischemic stroke: is gender important? J Cereb Blood Flow Metab. 2013;33(9):1355-1361.

62. Koerner IP, Gattimng M, Noppens R, et al. Induction of cerebral ischemic tolerance by erythromycin preconditioning reprograms the transcriptional response to ischemia and suppresses inflammation. Anesthesiology. 2007;106(3):538-547.

63. Kouchoukos NT, Masetti P, Murphy SF. Hypothermic cardiopulmonary bypass and circulatory arrest in the management of extensive thoracic and thoracoabdominal aortic aneurysms. Semin Thorac Cardiovasc Surg. 2003;15(4):333-339.
64. Nathan HJ, Rodriguez R, Wozny D, et al. Neuroprotective effect of mild hypothermiain patients undergoing coronary artery surgery with cardiopulmonary bypass: five-year follow-up of a randomizedtrial. $J$ Thorac Cardiovasc Surg. 2007;133(5):1206-1211.

65. Newman MF, Kirchner JL, Phillips-Bute B, et al. Longitudinal assessment of neurocognitive function after coronary-arterybypass surgery. $N$ Engl J Med. 2001;344(6):395-402.

66. Roof Rl, Hall ED. Gender differences in acute CNS trauma and stroke: neuroprotective effects of estrogen and progesterone. J Neurotrauma. 2000;17(5):367-381 